\title{
FOSTER-TYPE CRITERIA FOR MARKOV CHAINS ON GENERAL SPACES
}

\author{
BRIAN H. FRALIX, ${ }^{*}$ Georgia Institute of Technology
}

\begin{abstract}
This paper establishes new Foster-type criteria for a Markov chain on a general state space to be Harris recurrent, positive Harris recurrent, or geometrically ergodic. The criteria are based on drift conditions involving stopping times rather than deterministic steps. Meyn and Tweedie (1994) developed similar criteria involving random-sized steps, independent of the Markov chain under study. They also posed an open problem of finding criteria involving stopping times. Our results essentially solve that problem. We also show that the assumption of $\psi$-irreducibility is not needed when stating our drift conditions for positive Harris recurrence or geometric ergodicity.
\end{abstract}

Keywords: Markov chain; Foster's criterion; Harris recurrence; geometric ergodicity; drift criterion

2000 Mathematics Subject Classification: Primary 60J10

\section{Introduction}

The classical Foster criterion is well known for verifying whether an irreducible Markov chain on a countable state space is positive recurrent. Intuitively, this criterion assumes that the chain tends to drift (in unit steps) towards some small subset of the state space, and the chain does not wander too far when it makes a one-step transition out of this set.

This paper addresses the following issue. Can we find analogous drift criteria for Markov chains on general state spaces that are based on steps that may be larger than one or on random steps? Specifically, are there drift criteria that are sufficient for Harris recurrence, positive Harris recurrence, or geometric ergodicity?

The first study that addressed such issues was Filonov [3] (see also [7]). Filonov gave a sufficient drift condition for a Markov chain on a countable space to be ergodic for steps that are stopping times. Meyn and Tweedie [6] obtained similar results for Markov chains on arbitrary state spaces for deterministic steps. They also derived a sufficient condition involving steps that are conditionally independent of the Markov chain (given a fixed initial state), with tail probabilities that satisfy a certain property. Because of this property, their random statedependent drift criterion is not a generalization of their deterministic version.

In this paper, we present new Foster-type drift conditions involving steps that are stopping times with respect to a filtration that preserves the Markovian property of the Markov chain under study. In Theorem 3, we provide a sufficient condition for a Markov chain on a general state space to be Harris recurrent. It is based on a drift condition for steps that are stopping times with respect to a suitable filtration. Theorems 2.1(i) and 2.2(i) in [6] are special cases. Our next result (Theorem 4) is a sufficient condition for positive Harris recurrence under drift conditions for steps that are integrable stopping times. Theorem 4 generalizes many known

Received 20 February 2006; revision received 9 July 2006.

* Postal address: School of Industrial and Systems Engineering, Georgia Institute of Technology, Atlanta, GA 303320205, USA. Email address: bfralix@isye.gatech.edu 
results, including Filonov's result [3] (for a countable state space), [6, Theorem 2.1(ii)] (for deterministic steps), and [4, Theorem 1]. However, our Theorem 4 and [6, Theorem 2.1(ii)] are not comparable for some cases; see the example in [6, Section 2] for further insight into this. Our next result (Theorem 5) gives a sufficient condition for a Markov chain to be geometrically ergodic. The special case of this result for deterministic steps is [6, Theorem 2.1(iii)]. We conclude by giving another state-dependent drift condition for geometric ergodicity that is similar to [4, Theorem 1]. This result also generalizes [6, Theorem 2.1(iii)], but in a different way.

An important feature of our Theorems 4, 5, and 6 for establishing positive Harris recurrence and geometric ergodicity is that they do not require the Markov chain to be $\psi$-irreducible. Costa and Dufour [1] also showed that the $\psi$-irreducibility assumption is not needed for the one-step drift condition for positive Harris recurrence. Another subtlety in their drift condition is that it uses extended real-valued test functions - our conditions, and those in [6], simply use real-valued test functions.

\section{Preliminaries}

We will study the type of Markov chain discussed in [5]. Let $X:=\left\{X_{n}\right\}_{n=0}^{\infty}$ be a Markov chain with respect to a filtration $\mathcal{F}:=\left\{\mathcal{F}_{n}\right\}_{n=0}^{\infty}$ on an arbitrary state space $\mathbb{E}$ equipped with a countably generated $\sigma$-field $\mathscr{E}$ (for example, $\mathbb{E}$ could be a Polish space equipped with its Borel $\sigma$-field $\mathcal{E})$. The underlying probability space for the process is $(\Omega, \mathcal{A}, \mathrm{P})$.

We will frequently use stopping times of the form $\tau_{A}:=\inf \left\{n \geq 1: X_{n} \in A\right\}, A \in \mathcal{E}$. A set $C \in \mathcal{E}$ is petite if there exist a nontrivial measure $\mu$ on $\mathscr{E}$ and a probability measure $\alpha$ on the nonnegative integers such that

$$
K_{\alpha}(x, B):=\sum_{n=0}^{\infty} \alpha(n) \mathrm{P}_{x}\left(X_{n} \in B\right) \geq \mu(B), \quad x \in C, B \in \mathcal{E} .
$$

The Markov chain $X$ is $\psi$-irreducible if there exists a nontrivial measure $\psi$ on $\&$ such that $\mathrm{P}_{x}\left(\tau_{A}<\infty\right)>0, x \in \mathbb{E}$, for any $A \in \mathcal{E}$ with $\psi(A)>0$. The Markov chain $X$ is Harris recurrent if it is $\psi$-irreducible and $\mathrm{P}_{x}\left(\tau_{A}<\infty\right)=1, x \in A$, for any $A \in \mathcal{E}$ with $\psi(A)>0$.

A measure $\pi$ is invariant for $X$ if $\pi(A)=\int_{E} \mathrm{P}_{x}\left(X_{1} \in A\right) \pi(\mathrm{d} x), A \in \mathcal{E}$. A Harris recurrent Markov chain has a unique invariant measure (up to constant multiple), and the chain is positive Harris recurrent if the measure is finite.

The Markov chain $X$ is geometrically ergodic if there exists a function $M: E \rightarrow \mathbb{R}_{+}$and a $\rho<1$ such that

$$
\sup _{A \in \mathscr{E}}\left|\mathrm{P}_{x}\left(X_{n} \in A\right)-\pi(A)\right| \leq M(x) \rho^{n}, \quad n \geq 1 .
$$

We will use the following results from [6].

Theorem 1. Suppose that the Markov chain X is $\psi$-irreducible.

(i) ([6, Theorem 3.1(i)].) The Markov chain $X$ is Harris recurrent if there is a petite set $C \in \mathcal{E}$ such that $\mathrm{P}_{x}\left(\tau_{C}<\infty\right)=1$, for all $x \in \mathbb{E}$.

(ii) ([6, Theorem 3.2(i)].) The Markov chain $X$ is positive Harris recurrent if and only if there is a petite set $C \in \mathcal{E}$ such that $\mathrm{P}_{x}\left(\tau_{C}<\infty\right)=1$, for all $x$, and $\sup _{x \in C} \mathrm{E}_{x}\left[\tau_{C}\right]<\infty$.

(iii) ([6, Theorem 3.3(ii)].) The Markov chain X is geometrically ergodic if it is aperiodic and there exist a petite set $C \in \mathcal{E}$ and $a \kappa>1$ such that $\mathrm{E}_{x}\left[\kappa^{\tau_{C}}\right]<\infty$, for all $x$, and $\sup _{x \in C} \mathrm{E}_{x}\left[\kappa^{\tau_{C}}\right]<\infty$. 
Theorem 2. ([6, Theorem 3.1(ii)].) Suppose that $X$ is $\psi$-irreducible. There is a set $N \in \mathcal{E}$ such that $N^{\mathrm{c}}$ (the complement of $N$ ) is empty or absorbing, and $X$ restricted to $N^{\mathrm{c}}$ is Harris recurrent and $\psi(N)=0$. If $X$ is not Harris recurrent then $N$ is nonempty and, for any petite set $C \subseteq N$ and $x \in N$,

$$
\mathrm{P}_{x}\left(X_{n} \in N, n \geq 0\right)>0, \quad \mathrm{P}_{x}\left(X_{n} \in C \text { infinitely often }\right)=0 .
$$

\section{Results}

For the following results, we assume that the underlying probability space for the Markov chain $X$ has the additional structure that there is a shift operator $\theta: \Omega \rightarrow \Omega$ such that $X_{n}(\omega)=$ $X_{0}\left(\theta_{n} \omega\right)$, where $\theta_{0}=I$ (the identity mapping on $\Omega$ ) and $\theta_{n}=\theta \circ \theta_{n-1}, n \geq 1$. If $(\Omega, \mathcal{A})$ is the canonical probability space (i.e. the sequence space $\mathbb{E}^{\infty}$ equipped with the product $\sigma$-field), then $\theta$ is just the usual shift operator for sequences. by

Associated with a stopping time $\tau$, define random variables $0=\tau_{0}<\tau_{1}<\cdots$ on $(\Omega, \mathcal{A})$

$$
\tau_{n+1}=\tau_{n}+\tau \circ \theta_{\tau_{n}}, \quad n \geq 0 .
$$

Note that each $\tau_{n}$ is an $\mathcal{F}$-stopping time and, by the strong Markov property (which holds because our time index is discrete), the process $\bar{X}_{n}:=X_{\tau_{n}}, n \geq 0$, is also a Markov chain with respect to the filtration $\mathcal{F}^{\tau}:=\left\{\mathcal{F}_{\tau_{n}}\right\}_{n=0}^{\infty}$. In addition to the first entrance time $\tau_{A}$ to the set $A$, we will use

$$
\sigma_{A}:=\inf \left\{n \geq 0: X_{n} \in A\right\} \quad \text { and } \quad \bar{\sigma}_{C}:=\inf \left\{n \geq 0: \bar{X}_{n} \in C\right\}
$$

Our first result provides a sufficient condition for $X$ to be Harris recurrent.

Theorem 3. Suppose that the Markov chain $X$ is $\psi$-irreducible and there exist an $f: \mathbb{E} \rightarrow \mathbb{R}_{+}$ that is unbounded off petite sets, a finite $\mathcal{F}$-stopping time $\tau \geq 1$, and a petite set $C$ such that

$$
\mathrm{E}_{x}\left[f\left(X_{\tau}\right)\right] \leq f(x), \quad x \notin C .
$$

Then $X$ is Harris recurrent.

Proof. Proceeding as in the proof of [6, Theorem 2.1(i)], define $U_{n}=f\left(\bar{X}_{n}\right) \mathbf{1}_{\left\{\bar{\sigma}_{C} \geq n\right\}}$, where $\mathbf{1}_{\{\cdot\}}$ is the indicator function. Clearly, $\bar{\sigma}_{C}$ is an $\mathcal{F}^{\tau}$-stopping time. This fact, along with our drift condition (1) gives

$$
\mathrm{E}\left[U_{n} \mid \mathcal{F}_{\tau_{n-1}}\right]=\mathbf{1}_{\left\{\bar{\sigma}_{C} \geq n\right\}} \mathrm{E}\left[f\left(\bar{X}_{n}\right) \mid \mathcal{F}_{\tau_{n-1}}\right] \leq U_{n-1} .
$$

This implies that $U:=\left\{U_{n}\right\}_{n=0}^{\infty}$ is a nonnegative supermartingale with respect to the filtration $\mathscr{F}^{\tau}$, so it converges almost surely (a.s.) to a finite limit. Notice that if $\bar{\sigma}_{C}<\infty$ a.s., then the limit must be zero a.s.

Consider the set $N$ given in Theorem 2, and suppose that it is nonempty (otherwise we would be done). Notice that, while on the set $\Omega_{N}=\left\{X_{k} \in N, k \geq 0\right\}$, we only have to consider the case when $\lim _{n \rightarrow \infty} f\left(X_{n}\right)=\infty$ a.s. on $\Omega_{N}$, since $f$ is unbounded off petite sets and $\mathrm{P}_{x}\left(X_{n} \in G\right.$ infinitely often $)=0$ for any petite set $G \subseteq N$ and any $x \in N$, by Theorem 2 (if the limit inferior is finite with positive probability, then the contrapositive of the second part of Theorem 2 allows us to conclude that the chain is Harris recurrent). Therefore, $\lim _{n \rightarrow \infty} f\left(\bar{X}_{n}\right)=\infty$ a.s. on $\Omega_{N}$, which means that $\bar{\sigma}_{C}<\infty$ a.s. on $\Omega_{N}$ (notice the use 
of the supermartingale $U$ ). Since $\tau_{n}<\infty$ a.s. for each $n$, we also know that $\sigma_{C}<\infty$ a.s. on $\Omega_{N}$.

Now assume that $N^{\mathrm{c}}$ is nonempty (if it is empty, then our proof is complete by Theorem 1(i)). From Theorem 2, we know there is a petite set $D \subset N^{\mathrm{c}}$ such that $\psi(D)>0$, since $\psi(N)=0$. From Harris recurrence on $N^{\mathrm{c}}$, it follows that $\mathrm{P}_{x}\left(\sigma_{D}<\infty\right)=1, x \in N^{\mathrm{c}}$. However, for all paths not in $\Omega_{N}$, it follows that $\sigma_{D}<\infty$ a.s. for any initial point $y$, because $N^{\mathrm{c}}$ is an absorbing set.

From [5, Theorem 5.5.5], we know that $C \cup D$ is petite. Thus, for any $x \in \mathbb{E}$,

$$
\mathrm{P}_{x}\left(\sigma_{C \cup D}=\infty\right) \leq \mathrm{P}_{x}\left(\sigma_{C}=\infty, \Omega_{N}\right)+\mathrm{P}_{x}\left(\sigma_{D}=\infty, \Omega_{N}^{\mathrm{c}}\right)=0,
$$

which, using Theorem 1(i), completes the proof.

The following lemma will be used in the rest of our proofs.

Lemma 1. If there is a petite set $C$ such that $\mathrm{P}_{x}\left(\tau_{C}<\infty\right)>0$, for all $x \in \mathbb{E}$, then the Markov chain $X$ is $\psi$-irreducible.

Proof. Since $C$ is petite, there exist a probability measure $\alpha(\cdot)$ and a nontrivial measure $\psi$ such that

$$
K_{\alpha}(x, B) \geq \psi(B), \quad x \in C, B \in \mathcal{E} .
$$

Fix $y \in \mathbb{E}$. Since $\mathrm{P}_{y}\left(\tau_{C}<\infty\right)>0$, there exists an integer $n_{0}$ such that $\mathrm{P}_{y}\left(X_{n_{0}} \in C\right)>0$. Thus, for any set $B \in \mathcal{E}$ such that $\psi(B)>0$,

$$
\begin{aligned}
\sum_{m=n_{0}}^{\infty} \mathrm{P}_{y}\left(X_{m} \in B\right) & =\int_{\mathbb{E}}\left(\sum_{m=0}^{\infty} \mathrm{P}_{x}\left(X_{m} \in B\right)\right) \mathrm{P}_{y}\left(X_{n_{0}} \in \mathrm{d} x\right) \\
& \geq \int_{C} K_{\alpha}(x, B) \mathrm{P}_{y}\left(X_{n_{0}} \in \mathrm{d} x\right) \\
& \geq \psi(B) \mathrm{P}_{y}\left(X_{n_{0}} \in C\right) \\
& >0 .
\end{aligned}
$$

So, by [5, Proposition 4.2.1], $X$ is $\psi$-irreducible.

The following result is an extension of [4, Theorem 1] to the random-drift setting. Conditions (3)-(6), below, are similar to conditions (L1)-(L4) in [4].

Theorem 4. The Markov chain $X$ is positive Harris recurrent if there exist a petite set $C$, an $f: \mathbb{E} \rightarrow \mathbb{R}_{+}$bounded on $C$, constants $A$ and $\alpha$, a stopping time $\tau \geq 1$ a.s., and an $\mathcal{F}_{\infty}$-measurable random variable $\eta$ that satisfy the following:

$$
\begin{gathered}
\mathrm{E}_{x}\left[f\left(X_{\tau}\right)+\eta\right] \leq f(x), \quad x \notin C, \\
\sup _{x \in C} \mathrm{E}_{x}\left[f\left(X_{\tau}\right)+\eta\right]<\infty, \\
\eta(\omega) \geq \alpha>-\infty, \quad \omega \in \Omega, \\
\eta(\omega)>0, \quad X_{0}(\omega) \notin C, \\
\sup _{x \in C} \mathrm{E}_{x}[\tau]<\infty, \\
\tau(\omega) \leq A \eta(\omega), \quad X_{0}(\omega) \notin C .
\end{gathered}
$$


Theorem 4 is a generalization of many standard methods. When $\eta=\tau$, we have the general state space analogue of Filonov's result. In particular, if $\eta=\tau=g\left(X_{0}\right)$ for some function $g$ and $f$ is bounded on $C$, we end up with Meyn and Tweedie's criterion found in [6]. Another special case of Theorem 4 is Dai's method [2], which involves the use of fluid limits; see [4] for details on how this method is equivalent to satisfying certain drift criteria.

Proof of Theorem 4. In addition to our sequence $\left\{\tau_{n}\right\}_{n=0}^{\infty}$, we introduce another sequence, $\left\{\eta_{n}\right\}_{n=0}^{\infty}$, where $\eta_{0}=0$ and, for $n \geq 0, \eta_{n+1}=\eta_{n}+\eta \circ \theta_{\tau_{n}}$. Note that, by induction,

$$
\eta_{n}=\sum_{k=0}^{n-1} \eta \circ \theta_{\tau_{k}}
$$

Let $v:=\inf \left\{n \geq 0: \tau_{n} \geq \sigma_{C}\right\}$. This is an $\mathcal{F}^{\tau}$-stopping time since it is easy to see that $\{v \leq n\} \in \mathcal{F}_{\tau_{n}}$.

We will now show that $\left\{Y_{\nu \wedge n}\right\}_{n=0}^{\infty}$ is an $\mathcal{F}^{\tau}$-supermartingale, where $Y_{n}:=f\left(\bar{X}_{n}\right)+$ $\mathrm{E}\left[\eta_{n} \mid \mathcal{F}_{\tau_{n}}\right]$. Notice that, on the set $\{v>n\}$,

$$
\begin{aligned}
\mathrm{E}\left[Y_{n+1} \mid \mathcal{F}_{\tau_{n}}\right] & =\mathrm{E}\left[f\left(X_{\tau_{n}+\tau \circ \theta_{\tau_{n}}}\right)+\eta \circ \theta_{\tau_{n}} \mid \mathcal{F}_{\tau_{n}}\right]+\mathrm{E}\left[\eta_{n} \mid \mathcal{F}_{\tau_{n}}\right] \\
& =\mathrm{E}_{\bar{X}_{n}}\left[f\left(X_{\tau}\right)+\eta\right]+\mathrm{E}\left[\eta_{n} \mid \mathcal{F}_{\tau_{n}}\right] \\
& \leq f\left(\bar{X}_{n}\right)+\mathrm{E}\left[\eta_{n} \mid \mathcal{F}_{\tau_{n}}\right] \\
& =Y_{n} .
\end{aligned}
$$

Thus, $\left\{Y_{v \wedge n}\right\}_{n=0}^{\infty}$ is a nonnegative $\mathcal{F}^{\tau}$-supermartingale.

Since $f$ is nonnegative,

$$
\mathrm{E}_{x}\left[\eta_{\nu \wedge n}\right]=\mathrm{E}_{x}\left[\mathrm{E}_{x}\left[\eta_{\nu \wedge n} \mid \mathcal{F}_{\tau_{\nu \wedge n}}\right]\right] \leq \mathrm{E}_{x}\left[Y_{\nu \wedge n}\right] \leq f(x) .
$$

Using (7), we obtain

$$
\eta_{\nu \wedge n}=\sum_{k=0}^{n-1} \eta \circ \theta_{\tau_{k}} \mathbf{1}_{\{k<\nu\}} \geq A^{-1} \sum_{k=0}^{n-1} \tau \circ \theta_{\tau_{k}} \mathbf{1}_{\{k<\nu\}}=A^{-1} \tau_{\nu \wedge n} .
$$

The first inequality follows from (6), since $v>k$ implies that $X_{0}\left(\theta_{\tau_{k}} \omega\right) \notin C$. After taking limits, while using (8) and $\sigma_{C} \leq \tau_{\nu}$, we see that, for $x \notin C$,

$$
\mathrm{E}_{x}\left[\tau_{C}\right]=\mathrm{E}_{x}\left[\sigma_{C}\right] \leq A f(x) .
$$

For $x \in C$, we obtain

$$
\mathrm{E}_{x}\left[\tau_{C}\right]=\mathrm{E}_{x}\left[\tau_{C} \mathbf{1}_{\left\{\tau_{C} \leq \tau\right\}}\right]+\mathrm{E}_{x}\left[\tau_{C} \mathbf{1}_{\left\{\tau_{C}>\tau\right\}}\right]
$$

Now, by (9),

$$
\begin{aligned}
\mathrm{E}_{x}\left[\tau_{C} \mathbf{1}_{\left\{\tau_{C}>\tau\right\}}\right] & \leq \mathrm{E}_{x}\left[\tau \mathbf{1}_{\left\{\tau_{C}>\tau\right\}}\right]+\mathrm{E}_{x}\left[\mathbf{1}_{\left\{\tau_{C}>\tau\right\}} \mathrm{E}_{\bar{X}_{1}}\left[\tau_{C}\right]\right] \\
& \leq \mathrm{E}_{x}\left[\tau \mathbf{1}_{\left\{\tau_{C}>\tau\right\}}\right]+\mathrm{E}_{x}\left[\mathbf{1}_{\left\{\tau_{C}>\tau\right\}} A f\left(\bar{X}_{1}\right)\right] \\
& \leq \mathrm{E}_{x}\left[\tau \mathbf{1}_{\left\{\tau_{C}>\tau\right\}}\right]+A \mathrm{E}_{x}\left[f\left(\bar{X}_{1}\right)+\eta-\eta\right] .
\end{aligned}
$$

Using this with (2), (5), and (10), we have

$$
\mathrm{E}_{x}\left[\tau_{C}\right] \leq \mathrm{E}_{x}[\tau]+A \mathrm{E}_{x}\left[f\left(\bar{X}_{1}\right)+\eta\right]-A \alpha<\infty .
$$

Then $X$ is positive Harris recurrent by Lemma 1 and Theorem 1(i). 
Our next result provides sufficient conditions for $X$ to be geometrically ergodic.

Theorem 5. The Markov chain $X$ is geometrically ergodic if it is aperiodic and there exist an $f: \mathbb{E} \rightarrow[1, \infty)$, an $\mathcal{F}$-stopping time $\tau \geq 1$, a petite set $C$, and a constant $\kappa>1$ such that

$$
\begin{aligned}
& \mathrm{E}_{x}\left[\kappa^{\tau} f\left(X_{\tau}\right)\right] \leq f(x), \quad x \notin C, \\
& \sup _{x \in C} \mathrm{E}_{x}\left[\kappa^{\tau} f\left(X_{\tau}\right)\right]<\infty, \quad x \in C .
\end{aligned}
$$

Proof. This proof is similar to the proof of Theorem 4. Let $Y_{n}:=\kappa^{\tau_{n}} f\left(X_{\tau_{n}}\right)$. Then, on the set $\{v>n\}$,

$$
\begin{aligned}
\mathrm{E}\left[Y_{n+1} \mid \mathcal{F}_{\tau_{n}}\right] & =\mathrm{E}\left[\kappa^{\tau_{n}+\tau \circ \theta_{\tau_{n}}} f\left(X_{\tau_{n}+\tau \circ \theta_{\tau_{n}}}\right) \mid \mathcal{F}_{\tau_{n}}\right] \\
& =\kappa^{\tau_{n}} \mathrm{E}_{X_{\tau_{n}}}\left[\kappa^{\tau} f\left(X_{\tau}\right)\right] \\
& \leq Y_{n} .
\end{aligned}
$$

This shows that $\left\{Y_{v \wedge n}\right\}_{n=0}^{\infty}$ is a nonnegative supermartingale with respect to $\mathcal{F}^{\tau}$. Next, observe that

$$
\mathrm{E}_{x}\left[\kappa^{\tau_{\nu \wedge n}}\right] \leq \mathrm{E}_{x}\left[Y_{\nu \wedge n}\right] \leq \mathrm{E}_{x}\left[Y_{0}\right]=f(x), \quad x \in \mathbb{E} .
$$

Letting $n \rightarrow \infty$ and applying the monotone convergence theorem, we have

$$
\mathrm{E}_{x}\left[\kappa^{\sigma_{C}}\right] \leq \mathrm{E}_{x}\left[\kappa^{\tau_{v}}\right] \leq f(x), \quad x \in \mathbb{E} .
$$

Then, for $x \notin C, \mathrm{E}_{x}\left[\kappa^{\tau_{C}}\right]=\mathrm{E}_{x}\left[\kappa^{\sigma_{C}}\right] \leq f(x)<\infty$ and, for $x \in C$, we obtain

$$
\mathrm{E}_{x}\left[\kappa^{\tau_{C}}\right] \leq \mathrm{E}_{x}\left[\kappa^{\tau} \mathrm{E}_{X_{\tau}}\left[\kappa^{\sigma_{C}}\right] \mathbf{1}_{\left\{\tau_{C}>\tau\right\}}+\kappa^{\tau} \mathbf{1}_{\left\{\tau_{C} \leq \tau\right\}}\right] \leq \mathrm{E}_{x}\left[\kappa^{\tau} f\left(X_{\tau}\right)\right]
$$

Combining these observations yields $\mathrm{E}_{x}\left[\kappa^{\tau_{C}}\right]<\infty$ for all $x \in \mathbb{E}$, so again Lemma 1 tells us that $X$ is $\psi$-irreducible. Therefore, $X$ is geometrically ergodic by Theorem 1(iii).

It is also worth pointing out that a 'supermartingale' approach helps to derive another drift condition for geometric ergodicity that is analogous to [4, Theorem 1].

Theorem 6. The Markov chain $X$ is geometrically ergodic if it is aperiodic and there exist a petite set $C$, functions $f: \mathbb{E} \rightarrow[1, \infty), g: \mathbb{E} \rightarrow\{1,2,3, \ldots\}$, and $h: \mathbb{E} \rightarrow(0, \infty)$, and constants $A>0$ and $\kappa>1$ that satisfy the following:

$$
\begin{gathered}
\mathrm{E}_{x}\left[\kappa^{h(x)} f\left(X_{g(x)}\right)\right] \leq f(x), \quad x \notin C, \\
\sup _{x \in C} \mathrm{E}_{x}\left[\kappa^{h(x)} f\left(X_{g(x)}\right)\right]<\infty, \\
g(x) \leq A h(x), \quad x \notin C, \\
\sup _{x \in C} g(x)<\infty .
\end{gathered}
$$

Proof. The proof of this result is very similar to the proofs of Theorems 4 and 5 .

Remark 1. Theorems 3, 4, and 5 should allow us in principle to use tools from optimal stopping theory (see [8]) to determine whether or not various Markov chains are stable. In [8], the following examples were studied in detail:

$$
\begin{gathered}
s(x)=\inf _{\tau} \mathrm{E}_{x}\left[f\left(X_{\tau}\right)\right], \\
s(x)=\inf _{\tau} \mathrm{E}_{x}\left[f\left(X_{\tau}\right)+\tau\right] .
\end{gathered}
$$


If we could guarantee the existence of an optimal stopping time $\tau^{*}$, then we would only have to show that $\left\{x: \mathrm{P}_{x}\left(\tau^{*}=0\right)>0\right\}$ is petite. To do this, we would have to find a nontrivial upper bound $h$ of the payoff function $s$ and show that $h(x)<f(x)$ for all $x$ outside a petite set.

Remark 2. It is well known that the converse to Theorem 4 is also true (let $\tau=1$, and consult [5, Chapter 11]). Analogously, here is a converse to Theorem 3.

Proposition 1. If the Markov chain $X$ is Harris recurrent, then there exist an $f: \mathbb{E} \rightarrow \mathbb{R}_{+}$ unbounded off petite sets, a finite stopping time $\tau \geq 1$, and a petite set $C$ such that, for $x \notin C$,

$$
\mathrm{E}_{x}\left[f\left(X_{\tau}\right)\right] \leq f(x) .
$$

Proof. By $\psi$-irreducibility, we know from [5, Theorem 5.5.5] that there exists an increasing sequence of sets $\left\{C_{n}\right\}$ where $C_{n}$ is petite for each $n$ and $\bigcup_{n=1}^{\infty} C_{n}=\mathbb{E}$. Let $r$ be an integer large enough that $\psi\left(C_{r}\right)>0$. Then $\tau_{C_{r}}<\infty$ almost surely, for any initial starting point $x \in \mathbb{E}$.

Now consider the real-valued function $f(x):=\min \left\{n \geq 1: x \in C_{n}\right\}, x \in \mathbb{E}$. Clearly $f$ is unbounded off petite sets. Moreover, $\mathrm{E}_{x}\left[f\left(X_{\tau_{C_{r}}}\right)\right] \leq r<f(x), x \notin C_{r}$.

\section{Acknowledgements}

The author would like to thank Professor Richard Serfozo for his insightful comments that significantly improved the style of this paper. He would also like to thank two referees for their comments and suggestions, which also had a significant impact on the paper (Theorem 4 in particular).

\section{References}

[1] Costa, O. L. V. and Dufour, F. (2005). A sufficient condition for the existence of an invariant probability measure for Markov processes. J. Appl. Prob. 42, 873-878.

[2] DAI, J. G. (1995). On positive Harris recurrence of multiclass queueing networks: a unified approach via fluid limit models. Ann. Appl. Prob. 5, 49-77.

[3] Filonov, Y. P. (1990). Criterion for ergodicity of homogeneous discrete Markov chains. Ukranian Math J. 41, $1223-1225$.

[4] Foss, S. and Konstantopoulos, T. (2004). An overview of some stochastic stability methods. J. Operat. Res. Soc. Japan 47, 275-303.

[5] Meyn, S. P. And Tweedie, R. L. (1993). Markov Chains and Stochastic Stability. Springer, London.

[6] Meyn, S. P. and Tweedie, R. L. (1994). State-dependent criteria for convergence of Markov chains. Ann. Appl. Prob. 4, 149-168.

[7] Robert, P. (2003). Stochastic Networks and Queues. Springer, Berlin.

[8] Shiryayev, A. N. (1978). Optimal Stopping Rules. Springer, New York. 